\title{
Higgs-boson production induced by bottom quarks
}

\author{
Eduard Boos \\ Skobeltsyn Institute of Nuclear Physics, Moscow State University, Moscow, Russia
}

Tilman Plehn

Theory Division, CERN, Geneva, Switzerland

(Received 16 May 2003; published 14 May 2004)

\begin{abstract}
Bottom-quark-induced processes are responsible for a large fraction of the CERN Large Hadron Collider (LHC) discovery potential, in particular, for supersymmetric Higgs bosons. Recently, the discrepancy between exclusive and inclusive Higgs boson production rates has been linked to the choice of an appropriate bottom factorization scale. We investigate the process kinematics at hadron colliders and show that it leads to a considerable decrease in the bottom factorization scale. This effect is the missing piece needed to understand the corresponding higher order results. Our results hold generally for charged and for neutral Higgs boson production at the LHC as well as at the Fermilab Tevatron. The situation is different for single top quark production, where we find no sizable suppression of the factorization scale. Turning the argument around, we can specify how large are the collinear logarithms that can be resummed using the bottom parton picture.
\end{abstract}

DOI: 10.1103/PhysRevD.69.094005

PACS number(s): 12.38.Bx, 12.60.Jv, 14.80.Cp

\section{HIGGS BOSONS AT THE LARGE HADRON COLLIDER}

The combined CERN $e^{+} e^{-}$collider LEP precision measurements [1] suggest the existence of a light Higgs boson. In the case of a single standard model Higgs boson the CERN Large Hadron Collider (LHC) promises multiple coverage for any Higgs boson mass, which will enable us to measure its different decay modes and extract the couplings [2]. For a supersymmetric Higgs sector this coverage has to rely on fewer Higgs boson decay channels [2,3]. This is a direct consequence of the structure of the Higgs sector: while the minimal supersymmetric standard model (MSSM) predicts a light Higgs boson, it also predicts an enhancement of the coupling to down-type fermions, at the expense of the branching fractions to gauge bosons. This enhancement is an outcome of the two Higgs doublet structure in the MSSM: one doublet is needed to give mass to up-type and the other to down-type fermions. The vacuum expectation values of the two doublets are different, parameterized by $\tan \beta$ $=v_{2} / v_{1}$. In addition to a light scalar Higgs boson, the two Higgs doublet model includes a heavy scalar, a pseudoscalar, and a charged Higgs boson. None of these additional particles has a mass bounded from above, apart from triviality or unitarity bounds.

Of course, observables linked to properties of a light Higgs boson can serve as a probe of whether a new scalar particle is indeed consistent with the standard model Higgs boson $[4,5]$. There is, however, only one way to conclusively tell the supersymmetric Higgs sector from its standard model counterpart: to discover the additional heavy Higgs bosons and determine their properties.

At the LHC, the possible enhancement of down-type fermion Yukawa couplings by powers of $\tan \beta$ can render the search for a heavy scalar and pseudoscalar Higgs boson promising. For small values of $\tan \beta$ the Yukawa coupling of the charged Higgs boson is governed by the top quark mass $m_{t} / \tan \beta$, whereas for larger values of $\tan \beta$ the bottom Yukawa coupling $m_{b} \tan \beta$ dominates. While the chances of finding a heavy Higgs boson with a small value of $\tan \beta$ at the LHC are rather slim, the discovery of all heavy Higgs scalars in the large $\tan \beta \gtrsim 10$ regime is likely. This reflects the fact that the reach of the LHC for charged and neutral Higgs bosons is to a large degree owed to scattering processes that involve incoming bottom quarks. The completely exclusive processes are

$$
g g \rightarrow \bar{b} t H^{-}, \quad g g \rightarrow \bar{b} b \Phi,
$$

where $\Phi=h^{0}, H^{0}, A^{0}$ denotes any neutral scalar Higgs boson. From an experimental point of view, the identification of these final state bottom jets is tedious, because the dominant contribution comes from phase space configurations where the incoming gluons split into two collinear bottom quarks. The bottom quark rapidity distribution peaks at rapidities around two and the transverse momentum distributions around the bottom quark mass. After adding in the efficiency for a bottom tag this becomes a heavy price to pay in the analysis. Therefore in particular in the case of heavy Higgs bosons one usually prefers to look for the more or less inclusive channels

$$
g b \rightarrow t H^{-}, \quad g b \rightarrow b \Phi, \quad b \bar{b} \rightarrow \Phi
$$

At this point we do not explicitly discuss the bottom-quarkinduced inclusive channels $b \bar{b} \rightarrow W^{+} H^{-}[6]$ and $b g \rightarrow t W^{-}$ [7], which are both known to next-to-leading order QCD, because their impact on the discovery potential of the LHC is not drastic. We emphasize, however, that our argument will hold for them the same way we apply it to the processes in Eq. (2).

All bottom-quark-inclusive channels suffer from an additional uncertainty: the choice of the factorization scale of the bottom parton. In contrast to the gluon density, the dependence of the bottom parton density on the factorization scale is large even for scales above $O(100 \mathrm{GeV})$. Recently, it has been observed in higher order calculations [6-10] that vary- 
ing the bottom factorization scale around a smaller central value yields a more stable perturbative behavior. The same choice of scales can resolve the discrepancy between the total inclusive and exclusive rates, which is most prominent for the production channel $b \bar{b} \rightarrow \Phi$. However, choosing scales according to perturbative behavior is difficult, because most processes include inherent cancellations between different contributions, and it is hard to define which contributions actually have to be stable. Instead of this somewhat soft argument, we will derive an appropriate bottom factorization scale from the kinematics of the exclusive production process. In the following two sections we first investigate charged Higgs boson production, because it involves only one incoming bottom quark and two heavy central decay products. In Sec. V we will then generalize our result to neutral Higgs boson production and compare it to single top quark production.

Conventions. Throughout this paper we show consistent leading order cross section predictions, including the respective one loop coupling constant, running heavy quark masses, and CTEQ parton densities. Unless stated otherwise, we assume $\tan \beta=30$ for all MSSM processes. The exclusive cross sections are quoted with a massive $4.6 \mathrm{GeV}$ bottom quark in the matrix element and the phase space, while the bottom Yukawa coupling is set to the running bottom quark mass.

\section{BOTTOM PARTON SCATTERING AT THE LARGE HADRON COLLIDER}

Heavy-flavor-induced search channels for supersymmetric Higgs bosons have been explored for many years [11]. To begin with the charged Higgs boson, three search modes have been investigated. (1) Charged Higgs bosons can be pair produced in a Drell-Yan type process, mediated by a weak interaction vertex [12]. Moreover, they can be pair produced at the tree level in bottom quark scattering [6] or through a one loop amplitude in gluon fusion [13]. (2) One charged Higgs boson can be produced together with a $W$ boson via scattering of two bottom quarks or in gluon fusion [14]. (3) The charged Higgs boson can be produced in association with a top quark, which seems to be the most promising search channel $[7,8,15,16]$. The charged Higgs boson can be detected either decaying to a top and a bottom quark [17] or decaying to a tau lepton and a neutrino [18]. Both LHC Collaborations have tried to reproduce these phenomenological analyses, most successfully in the case of the decay to tau leptons $[19,20]$. An important lesson to learn from the next-to-leading order QCD calculations $[7,8]$ is that using the bottom pole mass as the Yukawa coupling severely overestimates the rates, as one would expect from what we know about Higgs boson decays to bottom quarks. ${ }^{1}$

\footnotetext{
${ }^{1}$ We can also compare these next-to-leading supersymmetry QCD calculations $[7,8]$ to the usual heavy parton subtraction schemes which add the exclusive and the inclusive channels [11]: for large final state masses, the higher order calculation of the inclusive channel is just the perturbatively consistent extension of the latter.
}

The case of neutral scalar Higgs bosons in the MSSM has been discussed in similar detail. In the standard model the inclusive production process $b \bar{b} \rightarrow \Phi$ is a small correction to the inclusive gluon fusion channel [21]. In a supersymmetry context, and in particular for large $\tan \beta$, the bottom-quarkinduced process dominates gluon fusion $[2,10,22,23]$. The typical Higgs boson decays are the same as for a light standard model Higgs boson, except for heavy MSSM scalars, where decays to muon or tau pairs are most promising [24]. In the region with an intermediate pseudoscalar Higgs boson mass and strong mixing effects [3] one can still explore the tau decay modes if the mass splitting between the Higgs bosons is larger than a typical tau meson energy resolution of the order of $10-15 \mathrm{GeV}$. Additional problems occur in the so-called intense coupling regime [25], i.e. the region at large $\tan \beta$, where all three MSSM Higgs masses are close to each other and very close to the plateau mass value $M_{h}^{\max }$ $\simeq M_{H}^{\min }$. The mass splitting becomes too small to distinguish the invariant mass peaks in the tau decays. Because the two photon decay has an extremely small branching fraction and the Yukawa couplings to bottom quarks for all three Higgs bosons are strongly enhanced, the most promising way to search for and separate the Higgs states is the production process involving $b$ jets with the Higgs bosons decaying to muons [26].

Motivated by this vast number of analyses in the MSSM Higgs sector, we turn to the simplest process available: charged Higgs boson production in association with a top quark involves only one incoming bottom quark and is an appropriate starting point to understand the issue of bottom partons at the LHC. The features of the exclusive and the inclusive charged Higgs boson production processes

$$
g g \rightarrow \bar{b} t H^{-}, \quad g b \rightarrow t H^{-}
$$

have been investigated in detail in Ref. [8]. Let us briefly review the most important observations.

For reasons described above, the searches for charged Higgs bosons (decaying to tau leptons) at the LHC usually do not require the observation of a final state bottom quark. The exclusive process $g g \rightarrow \bar{b} t H^{-}$is then dominated by collinear splitting of one of the gluons into a bottom quark pair:

$$
\left.\frac{d \sigma}{d p_{T, b}}\right|_{\text {asympt }} \propto \frac{p_{T, b}}{p_{T, b}^{2}+m_{b}^{2}},\left.\quad \sigma\right|_{\text {asympt }} \propto \log \left[\left(\frac{p_{T, b}^{\max }}{m_{b}}\right)^{2}+1\right] .
$$

These logarithms in the total cross section can be resummed, which is precisely the definition of bottom partons [11]. The factorization scale of the bottom partons is defined as the maximum transverse bottom quark momentum up to which the asymptotic form of the cross section is assumed to hold, and up to which the $\operatorname{logarithms} \log p_{T, b} / m_{b}$ are then resummed. This means that in Eq. (4) one can identify $p_{T, b}^{\max }$ with $\mu_{F, b}$. If we assume $p_{T, b}^{\max } \gg m_{b}$, which as we will see is true for all processes we consider, the numerical value for the bottom quark mass will have no impact on our argument-we could neglect it altogether $[8,9,11]$. The fac- 
torization scale is per se an artificial parameter, the dependence on which has to vanish after including all orders of perturbation theory. In the case of neutral Higgs boson production $b \bar{b} \rightarrow \Phi$ this has recently been demonstrated, including the next-to-next-to-leading order (NNLO) QCD corrections [10]. We are lucky in the case of bottom quarks: the comparison between the actual and the asymptotic forms of the exclusive cross section allows us to estimate $p_{T, b}^{\max }$ and therefore the bottom parton factorization scale for the inclusive process. This also tells us how large the "large logarithms," which are resummed using bottom parton densities, actually are.

There are two caveats to be kept in mind, though. First, to leading order the inclusive description $g b \rightarrow t H^{-}$neglects the transverse momentum of the bottom jet appearing in the exclusive process $g g \rightarrow \bar{b} t H^{-}$. For the two heavy states $t$ and $H^{-}$this is probably a good approximation, in particular after including detector effects for the top quark and Higgs boson decay products. Second, naive dimensional analysis suggests $\mu_{F, b}=m_{t}+m_{H}$, which is often used. This does not have to be correct. The only thing dimensional analysis tells us is that $\mu_{F, b} \propto\left(m_{t}+m_{H}\right)$, as long as the production process is dominated by the threshold region. It has been shown that the proportionality factor does not at all have to be unity $[8,9]$ and that indeed a wrong choice of scale leads to a systematic overestimate of the cross section [28], as is obvious from Eq. (4).

\section{ASYMPTOTIC BEHAVIOR IN THE BOTTOM QUARK VIRTUALITY}

To make our argument as transparent as possible, we will investigate the relation between the bottom factorization scale and the threshold mass in two steps. The threshold mass we will refer to as $M$; for the charged Higgs boson production process this means $M=m_{t}+m_{H}$. We can generally rewrite the exclusive and the inclusive production processes including one bottom parton as

$$
g g \rightarrow \bar{b} X_{M}, \quad b g \rightarrow X_{M},
$$

where $X_{M}$ denotes the heavy final state particles. For a typical gluon-induced LHC production process close to threshold we expect the invariant mass of the heavy system and the threshold mass $M$ to be similar. In the case of $g g \rightarrow \bar{b} t H^{-}$we investigate how close to threshold the production takes place in Fig. 1. The parameter $\Delta M$ is defined as the difference between the invariant mass of the heavy $t H^{-}$system and the threshold mass $M=m_{t}+m_{H}$. We indeed see that the distributions peak at small values $\Delta M / M \lesssim 1 / 8$, even though there are sizable tails toward larger invariant masses.

In the first step of our argument, we investigate the maximum value for the intermediate bottom quark virtuality $Q_{b}^{\max }$, up to which the asymptotic form of the exclusive cross section holds:

$$
Q_{b}^{\max }=C_{Q} M
$$

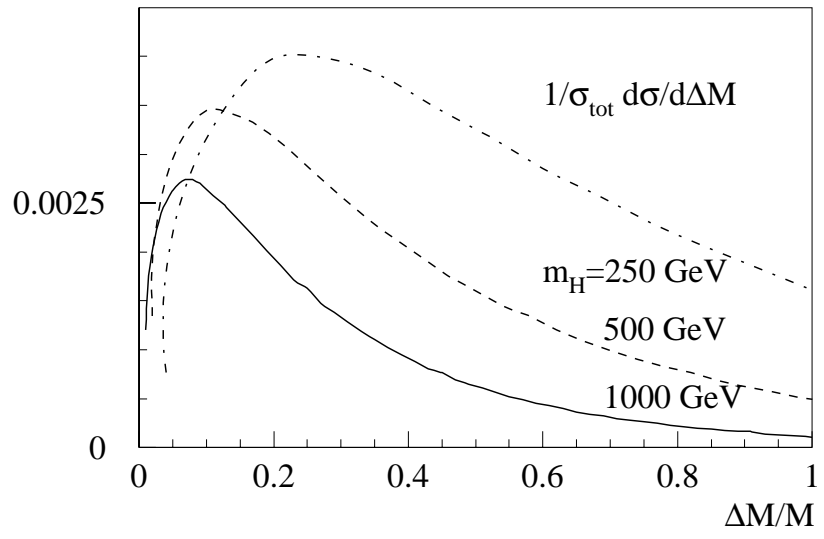

FIG. 1. Normalized $\Delta M$ distributions for the hadronic charged Higgs boson production $g g \rightarrow \bar{b} t H^{-}$, where $\Delta M$ is the difference between the invariant mass of the $t H^{-}$system and its threshold mass $M=m_{t}+m_{H}$.

In Sec. IV, we then estimate how far the asymptotic form in terms of the bottom transverse momentum is valid. From Ref. [8] we expect a relation $p_{T, b}^{\max } \sim M / 6$. If we assume that this reduction will consist of the suppression from the asymptotic behavior in the virtuality and an additional suppression when we move to the asymptotic behavior in the transverse momentum, we obtain

$$
\mu_{F, b} \equiv p_{T, b}^{\max }=C_{p} Q_{b}^{\max }=C_{p} C_{Q} M .
$$

To understand the asymptotic behavior of the hadronic cross section $\sigma\left(p p^{(-)} \rightarrow \bar{b} X_{M}\right)$ as a function of the bottom quark virtuality, we rewrite the integration over the phase space and the parton momentum fractions [9]. As long as we are interested in the behavior of the cross section for large values of the rapidity we can safely neglect the bottom quark mass:

$$
\begin{aligned}
\sigma= & \frac{1}{16 \pi} \frac{1}{S} \int_{0}^{S-M^{2}} d Q_{b}^{2} \int_{Q_{b}^{2}+M^{2}}^{S} d s \\
& \times \int_{(1 / 2) \log (s / S)}^{-(1 / 2) \log (s / S)} d y \mathcal{L}_{g g} \frac{1}{s^{2}} \overline{|\mathcal{M}|^{2}} .
\end{aligned}
$$

Here $Q_{b}$ is the intermediate bottom quark virtuality, $y$ the rapidity, and $\sqrt{S}$ and $\sqrt{s}$ are the hadronic and partonic collider energies. The factor $1 / s^{2}$ in the integrand is obvious from the difference in mass units between the matrix element and the partonic differential cross section $d \hat{\sigma} / d Q_{b}^{2}$, as it originally appears in the integral. The parton densities are denoted as $\mathcal{L}=P_{i / p}\left(x_{1}\right) P_{j / p}\left(x_{2}\right)$. At this point we make a few simplifying approximations: since we want to show that the asymptotic behavior in the virtuality is a processindependent phase space effect, we neglect all structures in the matrix element, except for the asymptotic behavior in the virtuality. The asymptotic form of the differential hadronic cross section $d \sigma / d Q_{b} \propto 1 / Q_{b}$ translates into $\overline{|\mathcal{M}|^{2}}$ $=S^{2} \sigma_{0} / Q_{b}^{2}$. The factor $S^{2}$ we introduce to absorb units of energy; it could as well be $M^{4}$. For reasons that will be 


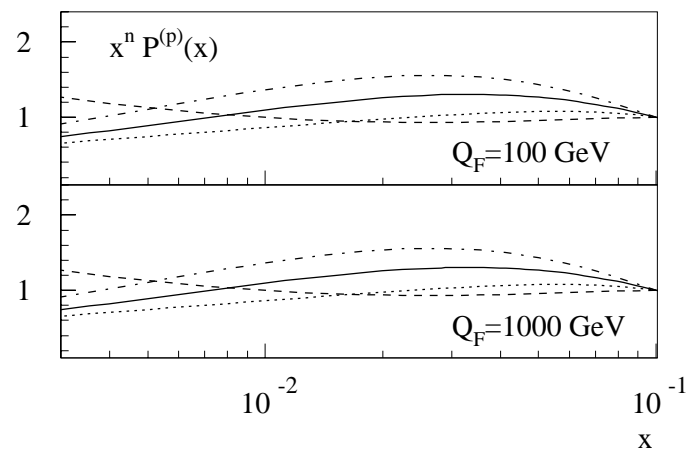

FIG. 2. Ratio of parton distributions in the proton and the function $x^{-n}$ for two different values of the factorization scale. All curves are normalized to their values at $x=0.1$. The different lines correspond to the gluon (solid, $n=2$ ), down-quark (dashed, $n$ $=1.1$ ), anti-up-quark (dotted, $n=1.7$ ), and bottom quark (dashdotted, $n=2$ ) content. We use CTEQ6L parton densities [29].

obvious later, it could not be a partonic variable, since we need to keep track of the powers of the parton momentum fraction. Furthermore, we assume that the steep gluon densities balance each other for the production of heavy states $x_{1}=x_{2}=\sqrt{x}$ for $x_{1}, x_{2} \ll 1$. The approximate hadronic cross section now reads

$$
\begin{aligned}
\sigma & =-\frac{2 \sigma_{0}}{16 \pi} \int_{0}^{S-M^{2}} \frac{d Q_{b}}{Q_{b}} \int_{\left(Q_{b}^{2}+M^{2}\right) / S}^{1} \frac{d x}{x^{2}} \mathcal{L}_{g g}(x) \log x \\
& =\frac{2 \sigma_{0} \mathcal{L}_{0}}{16 \pi} \int_{0}^{S-M^{2}} \frac{d Q_{b}}{Q_{b}} F\left(\tau\left(Q_{b}^{2}\right)\right)
\end{aligned}
$$

using

$$
\begin{aligned}
F(\tau) & =-\int_{\tau}^{1} \frac{d x}{x^{2}} \frac{1}{x^{j-2}} \log x \\
& =\frac{1}{(1-j)^{2}}\left[1-\tau^{1-j}+(1-j) \tau^{1-j} \log \tau\right] \sim Q_{b} \frac{d \sigma}{d Q_{b}},
\end{aligned}
$$

with $x=s / S$ and $\tau=\left(Q_{b}^{2}+M^{2}\right) / S$. The function $F(\tau)$ is a correction to the asymptotic behavior of the virtuality distribution $d \sigma / d Q_{b} \propto 1 / Q_{b}$. In an intermediate step we have approximated the incoming parton luminosity by a simple power suppression $\mathcal{L}=\mathcal{L}_{0} / x^{j-2}$. As a general parametrization of the parton densities, this is certainly not a good idea. However, looking at the production of heavy particles at the LHC we probe momentum fractions between $10^{-1}$ and few times $10^{-3}$. In Fig. 2 we show different parton distributions multiplied by $x^{n}$ for two values of the factorization scale. The ratio is normalized to its value at $x=0.1$. Looking at the different values of $n$ we see that our argument will at this point become dependent on the parton the incoming bottom parton sees on the other side. On the other hand, we also see that for $g g$ and $g b$ initial states the approximation $\mathcal{L}$ $\propto 1 /\left(x_{1} x_{2}\right)^{2}$ works very well. From Fig. 2 we obtain $j=4$ for

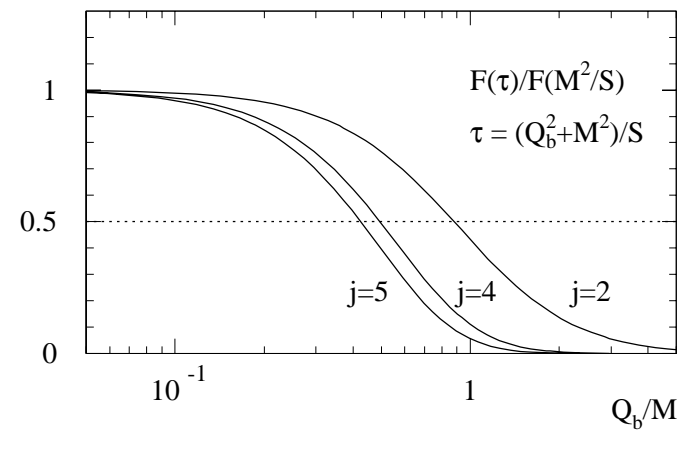

FIG. 3. The normalized function $F(\tau)$, defined in Eq. (10). The hadronic center-of-mass energy is set to $\sqrt{S}=14 \mathrm{TeV}$ and the threshold mass to $675 \mathrm{GeV}$, corresponding to a $500 \mathrm{GeV}$ charged Higgs boson. We display the behavior of the plateau in $Q_{b}$ for different values of $j$, which arise from the $x_{1} x_{2}$ behavior of the partonic cross section.

gluon or bottom initial states in the definition of $F(\tau)$. In Fig. 3 we show the behavior of $F(\tau)$ as a function of the bottom quark virtuality, the way it looks for a $500 \mathrm{GeV}$ charged Higgs boson at the LHC. The case of $j=2$ corresponds to constant parton densities $\mathcal{L} \equiv \mathcal{L}_{0}$.

Let us now turn to a detailed discussion of the function $F(\tau)$, shown in Fig. 3. As mentioned above, $F(\tau)$ is a correction to the known asymptotic behavior of the differential hadronic cross section with respect to the bottom quark virtuality. As in Ref. [8], we show the (normalized) curves for $Q_{b} d \sigma / d Q_{b}$, which we numerically obtain for the exclusive $\bar{b} t H^{-}$production process, in Fig. 4 . The first thing we notice from the exact results in Fig. 4 is that, as a function of $Q_{b} / M$, the curves for different Higgs boson masses are almost identical. The only major difference arises from the finite bottom mass effects, since their onset does not scale with $Q_{b} / M$, but with $m_{b} / Q_{b}$. On the other hand, these mass effects are understood and of no relevance to our argument, which is concerned with the upper end of the asymptotic behavior in $Q_{b}$. We roughly indicate this upper end of the plateau with a dotted line, where $Q_{b} d \sigma / d Q_{b}$ has dropped to half of its plateau value. In the first row in Fig. 4 we observe how the plateau in the virtuality does not at all extend to $Q_{b}=M$. The asymptotic approximation of the virtuality distribution is valid only up to values $Q_{b}^{\max } \sim M / 2.5$. In the second row we see that this picture changes when we ignore the gluon densities, but keep everything else, like in the complete numerical analysis: now the asymptotic behavior extends to $Q_{b} \gtrsim M$. In other words, the short plateau in $Q_{b} d \sigma / d Q_{b}$ is an effect of the steep gluon density in the proton. In the last row we also show how the approximation of the gluon luminosity $\mathcal{L}_{g g}\left(x_{1}, x_{2}\right) \sim 1 /\left(x_{1} x_{2}\right)^{2}$, which is a major ingredient we use to derive the approximate form $F(\tau)$, works very well for the virtuality distribution.

Since our main interest is the size of the bottom quark virtuality plateau, we need to compare the approximate form of $F(\tau)$ in Fig. 3 with the exact distribution $Q_{b} d \sigma / d Q_{b}$ in the first row of Fig. 4. In all figures we include the line which indicates where $Q_{b} d \sigma / d Q_{b}$ has decreased to half the plateau value. This is as good a measure for the extension of the 

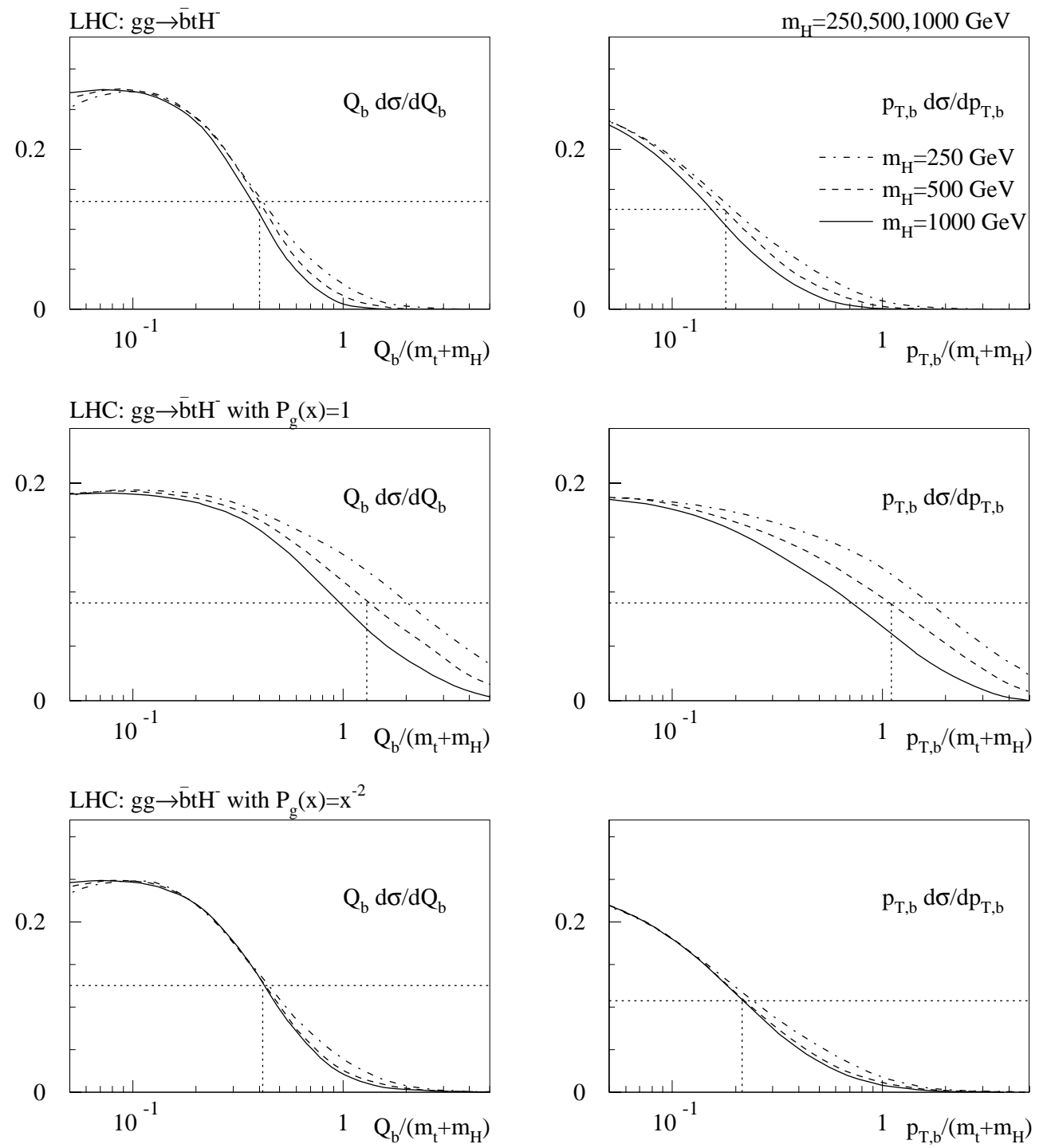

FIG. 4. Normalized distributions for the hadronic charged Higgs boson production process, for the complete gluon density (first row), for a constant gluon density (second row), and for the approximate gluon density $P(x)=1 / x^{2}$ (third row). The left column shows the bottom quark virtuality distribution, the right column the bottom quark transverse momentum. The normalization for the largest Higgs boson mass is by the total rate; for all other masses the curves are normalized such that their maxima coincide. The normalization factors for the virtuality and the transverse momentum are identical. We note that for a comparison with the approximate form $F(\tau)$ we have to identify $M=m_{t}$ $+m_{H}$.

plateau as any other. For the discussion of the approximate function $F(\tau)$ we prefer a better suited measure: the turning point of $F\left(\tau\left(\log Q_{b} / M\right)\right)$,

$$
\left.\frac{d^{2} F\left(\tau\left(Q_{b}^{2}\right)\right)}{d\left(\log Q_{b}^{2}\right)}\right|_{Q_{b}^{\max }}=0 .
$$

In Fig. 3 and Fig. 4 we see that this definition gives essentially the same $Q_{b}^{\max }$ values as the dotted line. The numerical values for $Q_{b}^{\max }$ which we compute from the definition of $F(\tau)$ are given in Table I for different values of $j$. The case $j=2$ corresponds to the case with constant parton densities. It is in very good agreement with what we see in the second row of Fig. 4 . The case $j=4$ should give the extension of the plateau for $g g$ - and $q b$-initiated processes, for example the charged Higgs boson production process. We see that the approximation $\overline{|\mathcal{M}|^{2}}=S^{2} \sigma_{0} / Q_{b}^{2}$ does not give a perfect prediction of $Q_{b}^{\max }$, as it leads to $Q_{b}^{\max } \sim M / 1.8$. For interfering $s$ and $t$ channel diagrams in the production process $g g$ $\rightarrow \bar{b} X_{M}$, the common denominator in the differential cross section typically becomes $1 /\left(s Q_{b}^{2}\right)$, while the numerator is dominated by the heavy mass. In our simple approximation we did not take into account this additional factor $1 / s$, which increases $j$ to 5 and moves the turning point to $Q_{b}^{\max } \lesssim M / 2$ and therefore much closer to the values seen in Fig. 4.

To summarize this section, we have shown that partonic phase space effects are responsible for the maximum value $Q_{b}^{\max }$ up to which the asymptotic behavior of the hadronic 
TABLE I. Maximum values for the bottom quark virtuality at the Tevatron and at the LHC, as defined in Eq. (10) and Eq. (11). The values of $j$ correspond to the power of $x$ in Eq. (10), as it arises from the $x_{1} x_{2}$ behavior of the partonic cross section.

\begin{tabular}{lccccccc}
\hline \hline$\sqrt{S}$ & $M$ & $Q_{b}^{\max }$ & $Q_{b}^{\max } / M(j=4)$ & $Q_{b}^{\max }$ & $Q_{b}^{\max } / M(j=5)$ & $Q_{b}^{\max }$ & $Q_{b}^{\max } / M(j=2)$ \\
\hline 2000 & 130 & 74 & $1 / 1.76$ & 64 & $1 / 2.03$ & 123 & $1 / 1.06$ \\
& 250 & 142 & $1 / 1.76$ & 123 & $1 / 2.03$ & 235 & $1 / 1.06$ \\
& 500 & 282 & $1 / 1.77$ & 245 & $1 / 2.04$ & 463 & $1 / 1.08$ \\
& 1000 & 559 & $1 / 1.79$ & 488 & $1 / 2.05$ & 908 & $1 / 1.10$ \\
14000 & 130 & 72 & $1 / 1.81$ & 64 & $1 / 2.03$ & 119 & $1 / 1.09$ \\
& 250 & 138 & $1 / 1.81$ & 121 & $1 / 2.07$ & 221 & $1 / 1.13$ \\
& 500 & 271 & $1 / 1.85$ & 238 & $1 / 2.10$ & 416 & $1 / 1.20$ \\
\hline \hline
\end{tabular}

cross section with respect to the bottom quark virtuality is valid. Our very simple approximation agrees with the numbers we obtain for the full hadronic process $g g \rightarrow \bar{b} t H^{-}$in Fig. 4:

$$
Q_{b}^{\max }=C_{Q} M,\left.\quad C_{Q}\right|_{\text {approx }} \sim \frac{1}{2},\left.\quad C_{Q}\right|_{b t H} \sim \frac{1}{2.5} .
$$

These values of $C_{Q}$ are of course linked to our definition of the collinear phase space region through the turning point of the function $F(\tau)$. However, comparing the behavior of the approximate function $F(\tau)$ in Fig. 3 and the exact exclusive cross section in Fig. 4 we see that any criterion will give compatible approximate and exact values of $C_{Q}$. One example is the end of the plateau as defined in Ref. [9] which gives a smaller value of $C_{Q}$.

\section{ASYMPTOTIC BEHAVIOR IN THE BOTTOM QUARK TRANSVERSE MOMENTUM}

From the discussion of the bottom quark virtuality one would expect to be able to follow a similar set of arguments for the bottom quark transverse momentum. Unfortunately, the phase space parametrization reflects the fact that the hadronic cross section factorizes in the virtuality and not in the transverse momentum. Instead, we choose a different path: we know that the asymptotic behavior $d \sigma / d p_{T, b} \propto 1 / p_{T, b}$ has to hold for small transverse momenta. In that regime the longitudinal momentum of the outgoing bottom quark in the center-of-mass system will be much larger than the transverse momentum: $\rho \equiv p_{z, b} / p_{T, b} \gg 1$. On the other hand, we want to push this approximation to its limits, $\rho \ll 1$. The general relation between the virtuality and the transverse momentum of the bottom jet for the production of a heavy system $X_{M}$ at threshold is

$$
\frac{Q_{b}^{2}}{M^{2}}=\frac{p_{T, b} \sqrt{s}}{M^{2}}\left(\sqrt{1+\rho^{2}}-\rho\right) \sim \frac{p_{T, b}}{M} .
$$

In the intermediate step we have assumed that in the limit of large bottom quark virtuality and large transverse momentum the bottom jet has dominantly a transverse momentum direction, i.e. that the bottom jet is central in the detector. We now require that the phase space region which forms the upper end of the plateau in the virtuality $\left(Q_{b}^{\max }\right)$ is also responsible for the upper end of the transverse momentum plateau $\left(p_{T, b}^{\max }\right)$. From the approximate result in Eq. (12) we obtain

$$
\mu_{F, b}=p_{T, b}^{\max }=C_{p} Q_{b}^{\max }=C_{p} C_{Q} M,\left.\quad C_{p}\right|_{\text {approx }}=\left.C_{Q}\right|_{\text {approx }}=\frac{1}{2} .
$$

To understand this effect in more detail, we now keep $\rho$ as a free parameter and assume that the heavy system $X_{M}$ is produced at threshold. We find

$$
p_{T, b}=\frac{s-M^{2}}{2 \sqrt{s} \sqrt{1+\rho^{2}}} .
$$

We can solve the equation for $\sqrt{s}$ and insert it into Eq. (13), which leaves us with

$$
\frac{Q_{b}^{2}}{M^{2}}=\frac{p_{T, b}^{2}}{M^{2}}\left(1+\rho^{2}-\rho \sqrt{1+\rho^{2}}\right)\left(1+\sqrt{1+\frac{M^{2}}{p_{T, b}^{2}\left(1+\rho^{2}\right)}}\right) .
$$

This function has two limiting cases. For small transverse momentum $(\rho \gg 1), p_{T, b}$ scales with the virtuality $p_{T, b}$ $\sim Q_{b}$. This means that the plateau in the transverse momentum will extend to the same value as the plateau in the virtuality. This observation suggests that (at least for not too large transverse momenta) the phase space region which dominates the high end of the plateau in $Q_{b}$ will also be responsible for the upper end of the plateau in $p_{T, b}$. We have confirmed this assumption for charged Higgs boson production explicitly. In contrast, for very large transverse momentum $(\rho \ll 1)$, a correction occurs to the linear relation between $p_{T, b}$ and $Q_{b}$. This correction pushes $p_{T, b}$ to smaller values, and the corrections becomes bigger for small values of $p_{T, b} / M$, which is what we expect from the shifted and softened transverse momentum plateau in Fig. 4.

To translate $Q_{b}^{\max }$ into $p_{T, b}^{\max }$ we evaluate Eq. (16) numerically. The left hand side we substitute by $C_{Q}$, which according to the previous section assumes numerical values of $1 / 2$ to $1 / 3$. Figure 5 shows the corresponding values of $C_{p}$ $=p_{T, b}^{\max } / Q_{b}^{\max }$ for different $\rho$ values. Indeed $C_{p}=1$ holds for small transverse momentum $\rho \gtrsim 5$. For all other values of $\rho$ the transverse momentum is always considerably smaller 


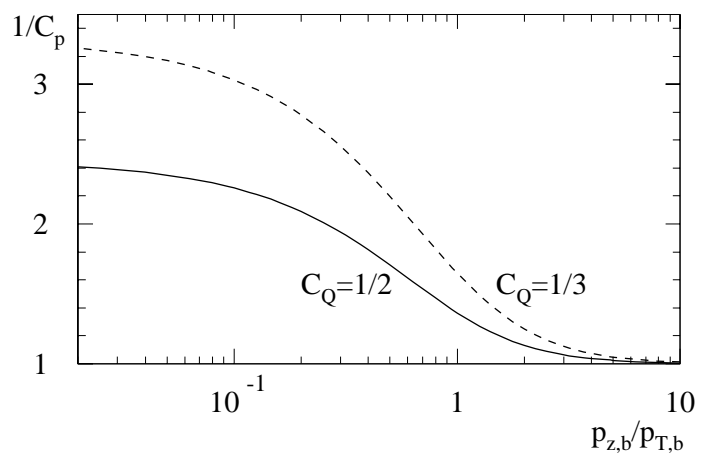

FIG. 5. The numerical solution of Eq. (16) for two different values of $C_{Q}=Q_{b} / M$, giving $C_{p}=p_{T, b} / Q_{b}$ as a function of $\rho$ $=p_{z, b} / p_{T, b}$. The longitudinal momentum of the bottom quarks is defined in the parton center-of-mass system.

than the virtuality. In other words: if we want the transverse momentum to be as large as possible for a given virtuality, we would have to make $\rho$ large, i.e. make the longitudinal momentum even larger. The limiting factor will be once more the steep gluon luminosity. In the opposite regime $\rho$ $\ll 1$, which will be preferred by the gluon density, we find a very substantial reduction factor $C_{p} \leqslant 1 / 2$.

In Fig. 6 we plot the $\rho$ distribution for exclusive charged Higgs boson production. It is clearly peaked at small values of $\rho$, which means large values of $p_{T, b}$. The peak becomes considerably more pronounced if we allow virtualities only above $M / 5$, which limits the phase space to the transition region in the virtuality plateau. According to Fig. 5, the region $\rho \ll 1$ leads to transverse momenta much smaller than the virtuality. Even though the actual distribution in Fig. 6 peaks at $\rho=0$ these events will no longer contribute to the upper end of the plateau in $p_{T, b}$; instead the plateau in $p_{T, b}$ will be softened. At the opposite end, $\rho \gtrsim 2$, we would expect a negligible suppression factor $C_{p}$ and large transverse momenta from virtualities around $Q_{b}^{\max }$, but the actual distribution shows how the gluon luminosities cut heavily into this region. What is left is the intermediate region $\rho$ $=0.5, \ldots, 1.5$, which interpolates between the extremes and

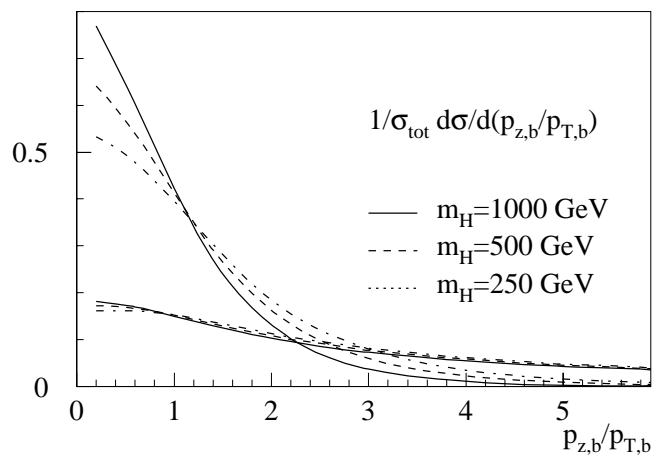

FIG. 6. Normalized $\rho$ distributions for the hadronic charged Higgs boson production $g g \rightarrow \bar{b} t H^{-}$. The parameter $\rho$ is the ratio of the longitudinal and transverse momentum of the bottom jet $\rho$ $=p_{z, b} / p_{T, b}$ in the parton center-of-mass system. The steeper set of curves is after a cut $Q_{b}>M / 5$. contributes most to the upper end of the plateau in $p_{T, b} d \sigma / d p_{T, b}$. Even though the numerical details used in this argument are not process independent, the fact that one has to interpolate between the two extreme regions in phase space is completely general.

This leaves us with two conclusions concerning the connection between the asymptotic regions in the bottom quark virtuality and transverse momentum. First, the plateau will not just translate from $Q_{b} d \sigma / d Q_{b}$ to $p_{T, b} d \sigma / d p_{T, b}$. Instead, it will be softened. Second, we can extract our approximate prediction for $C_{p}$ from Fig. 5 (keeping in mind $C_{Q} \sim 1 / 2.5$ ) and compare it with what we find in Fig. 4:

$$
\begin{gathered}
\mu_{F, b}=p_{T, b}^{\max }=C_{p} Q_{b}^{\max },\left.\quad C_{p}\right|_{\mathrm{approx}} \sim \frac{1}{1.4}, \cdots, \frac{1}{2}, \\
\left.C_{p}\right|_{b t H} \sim \frac{1}{2} .
\end{gathered}
$$

Combining these results with Sec. III we now understand that for charged Higgs boson production the plateau in $p_{T, b} d \sigma / d p_{T, b}$ does not extend to values $p_{T, b}^{\max } \sim M$. Making use essentially of phase space effects we instead find $p_{T, b}^{\max }$ $\sim M / 4$. This result confirms the value $p_{T, b}^{\max } \sim M / 5$, which we find directly from the exclusive process $g g \rightarrow \bar{b} t H^{-}$[8], which implies that using the naive bottom quark factorization scale $\mu_{F, b}=M$ will overestimate the cross section considerably. On the other hand, higher order QCD corrections [6-10] soften the dependence on the factorization scale considerably.

We can also turn this argument around: the bottom parton approach means integrating over the additional bottom quark phase space and resumming the logarithms including the transverse momentum. Going back to Eq. (4), we see that the terms which we resum are at maximum $\alpha_{s} \log \left[\left(p_{T, b}^{\max } / m_{b}\right)^{2}\right]$. For a charged Higgs boson of mass $500 \mathrm{GeV}$ this gives $6.7 \alpha_{s}$. For a threshold mass $M=130 \mathrm{GeV}$ the logarithm yields only moderate $3.5 \alpha_{s}$, using $p_{T, b}^{\max }=M / 5$.

\section{SIMILAR AND NOT SO SIMILAR PROCESSES}

\section{A. Neutral Higgs boson production}

In the previous sections we derived the appropriate bottom parton factorization scale for the associated production of a charged Higgs boson and a top quark. For different reasons this process is particularly well suited for the bottom parton description: there is only one bottom parton, the topquark-Higgs-boson system is very heavy, it will be produced close to threshold, and (for those reasons) it will be slow moving and central in the detector. A process that is particularly important for light as well as for heavy supersymmetric Higgs bosons is the production of a neutral scalar in association with two bottom quarks, which at the different level of inclusive versus exclusive description reads $[22,10]$

$$
g g \rightarrow \bar{b} b \Phi, \quad b g \rightarrow b \Phi, \quad b \bar{b} \rightarrow \Phi .
$$



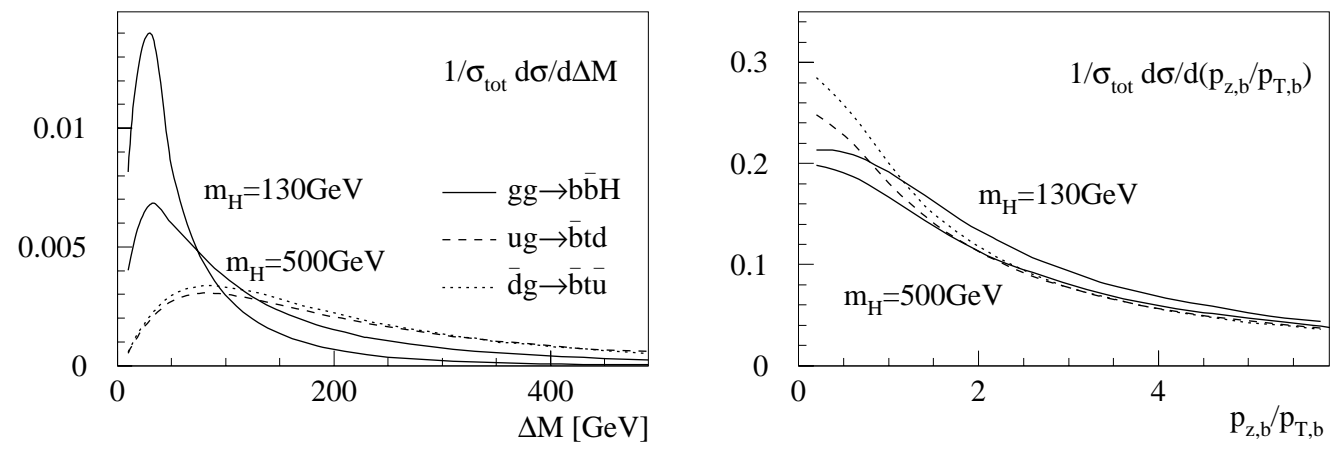

FIG. 7. Normalized distributions for hadronic neutral Higgs boson production $g g \rightarrow \bar{b} b \Phi$ and single top quark production $q g \rightarrow \bar{b} t q^{\prime}$. Left: difference between the invariant mass of the heavy system and its threshold mass. Right: ratio of the longitudinal and transverse momenta of the bottom jet $\rho=p_{z, b} / p_{T, b}$ in the parton center-of-mass system.

A phenomenological interesting aspect is that these kind of channels with at least one tagged bottom jet $[9,27]$ can prove an enhanced bottom Yukawa coupling. We know that just as in the charged Higgs boson case, the factorization scale of the bottom parton has to be chosen well below $m_{\Phi}$ $[8,9]$. The first reason is that again a heavy system $\left(X_{M}\right.$ $=b \Phi)$ is produced close to threshold, Fig. 7. In Fig. 8 we show the plateau in $Q_{b} d \sigma / d Q_{b}$, similarly to the figures in Ref. [9], and obtain $Q_{b}^{\max } \sim M / 2.5$. As for the charged Higgs boson case we see that the curves are nearly degenerate for different Higgs boson masses, i.e. $Q_{b}^{\max } \propto M$. For the neutral Higgs bosons an additional curve is included in Fig. 8, assuming a $130 \mathrm{GeV}$ light scalar MSSM Higgs boson. In this case the plateau is not particularly wide, since the bottom mass effects bend down the curves at fairly large values of $Q_{b} / M$. On the other hand, these effects are understood, so that the altered shape can be treated just like a plateau. Moreover, the dominant effects will arise from the upper end of the curve, where the logarithms are largest.

Comparing Fig. 4 with Fig. 8, we see that the extension of the asymptotic behavior in the bottom quark virtuality at the LHC is just the same for charged Higgs boson production $\left(b g \rightarrow t H^{-}\right)$as for neutral Higgs boson production ( $b g$ $\rightarrow b \Phi)$, for similar heavy state masses. An aspect we did not discuss in the charged Higgs boson case is that the plateau seems to extend to slightly larger virtualities for smaller threshold masses, in particular for a $130 \mathrm{GeV}$ neutral Higgs boson. The reason is that, in general, $X_{M}$ will be produced relatively closer to threshold for heavier states, i.e. $\Delta M / M$ becomes smaller, even though Fig. 1 and Fig. 7 show that in absolute numbers $\Delta M$ becomes slightly larger for heavier states $X_{M}$.

Up to this point we did not have to specify the collider energy in our approximation. Moreover, charged Higgs boson production is relevant only at the LHC. The distributions for neutral Higgs boson production at the Tevatron are given in Fig. 9. While for small Higgs boson masses of $130 \mathrm{GeV}$ the bottom quark virtuality plateau looks very similar to corresponding curves for the LHC, our picture starts to break down for very large Higgs boson masses. In that case the limited hadronic collider energy does not allow production of heavy states plus a bottom jet with sizable virtuality/ transverse momentum. Of course, the case of a $500 \mathrm{GeV}$
Higgs boson at the Tevatron is phenomenologically irrelevant. On the other hand, we learn that implicitly we have used another approximation in our discussion of the LHC processes: that the production rate even for large virtuality is limited only by the parton densities and the parton energy, never by the hadronic collider energy. Implicitly we check this requirement in Fig. 4, when we confirm $Q_{b}^{\max } \sim M$ after neglecting the effect of parton densities.

Last but not least, the question is what will happen for the completely inclusive Higgs boson production $(b \bar{b} \rightarrow \Phi)$ ? From Ref. [9] we know that the argument for the plateau in $Q_{b} d \sigma / d Q_{b}$ works just the same way as before, except that we now use the semi-exclusive process $b g \rightarrow b \Phi$ to compute $Q_{b}^{\max }$. Using the approximation described in Sec. III we understand what happens: the mass of the heavy system is still $M=m_{\Phi}$, but the gluon that splits into a bottom quark pair now sees a bottom parton on the other side instead of the gluon in the completely exclusive process. In Fig. 2 we see that the approximations $\mathcal{L}_{b g} \sim 1 /\left(x_{1} x_{2}\right)^{2}$ and $x_{1} \sim x_{2}$ still work fine. In a way this is a consistency check, of course. A significant change in the behavior of the bottom partons between the first bottom jet and the second bottom jet to be integrated over in the process $g g \rightarrow b \bar{b} \Phi$ would pose a serious problem for factorization in general. This result is in very good agreement with the results of the NNLO QCD calculation for this process: the small bottom quark factorization scale indeed yields perturbative corrections which are well under control [10].

The second step is again the transition from the plateau in virtuality to that in transverse momentum. Not surprisingly, from the exclusive process we numerically find the same behavior as for the charged Higgs boson, Fig. 4. Our argument in Sec. IV also works in just the same way for neutral Higgs bosons. From the exclusive process we numerically find $\mu_{F, b} \sim M / 5$, which we can understand in complete analogy to the charged Higgs boson case in the previous sections.

\section{B. Single top quark production}

In contrast to the Higgs boson production mechanisms, the bottom parton picture in single top quark production $[30,31]$ 

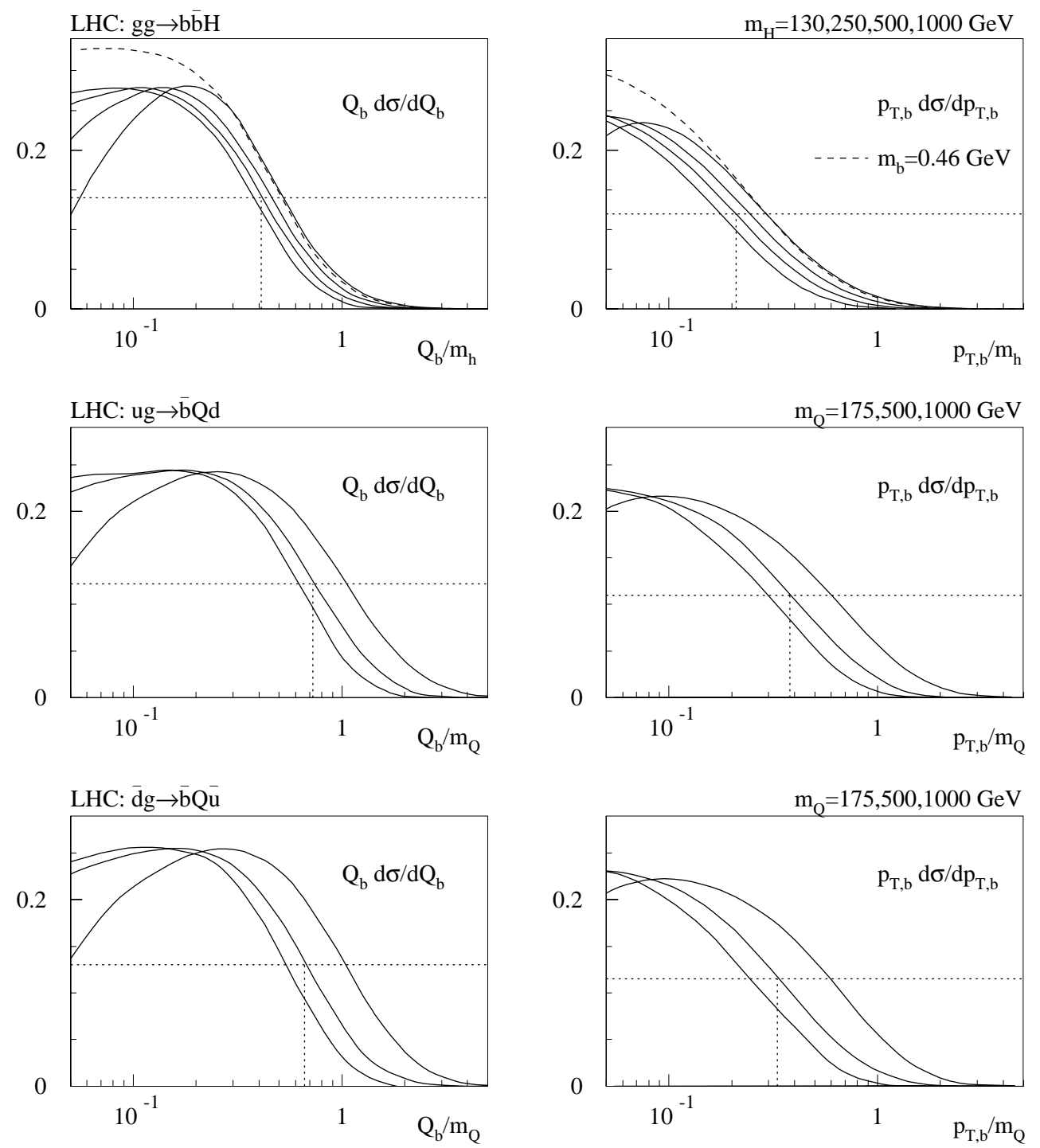

FIG. 8. Normalized distributions for the hadronic neutral Higgs boson production and for exclusive single top quark production at the LHC, where the varied mass of the generalized top quark is $m_{Q}$. The normalization for the largest Higgs boson mass is by the total rate; for all other masses the curves are normalized such that the virtuality distributions coincide at their maxima. The normalization factors for the virtuality and the transverse momentum are identical. The dashed curve uses a mathematical cutoff $0.46 \mathrm{GeV}$ for the bottom quark mass and $130 \mathrm{GeV}$ for the Higgs boson mass. It is normalized to match the curve for the physical bottom quark mass for large virtuality. The curves are ordered on their downward slopes by decreasing final state mass toward larger values of $Q_{b}^{\max }$ and $p_{T, b}^{\max }$.

$$
u g \rightarrow \bar{b} t d \quad(u b \rightarrow t d), \quad \bar{d} g \rightarrow \bar{b} t \bar{u} \quad(\bar{d} b \rightarrow t \bar{u})
$$

has never posed a problem. The difference between the two above processes is that the first one will involve valence quarks at the LHC, while the second one will not. Looking back, Fig. 2 shows that these channels should look slightly different, if our argument in Sec. III is correct.

In the left column in Fig. 8 we see how the single top quark case differs from the Higgs boson production. First of all, the plateau in the virtuality extends considerably further, typically to $Q_{b}^{\max } \gtrsim M / 1.5$. This is in agreement with the less steep parton densities for the quarks, which the splitting gluon sees. If we take a closer look, we even see that the plateau extends further in the case of an incoming valence quark than for a sea quark, which is in agreement with the approximate parton densities, Fig. 2.

However, our approximation has to be looked at with some care, since now we cannot assume $x_{1} \sim x_{2}$ anymore. Furthermore, in Fig. 7 we see that the single top quark production does not happen at all close to threshold. It peaks around $\Delta M \sim m_{W}$, which reflects the fact that one could integrate over the phase space of the outgoing jet and regard the single top quark process as $b W$ scattering. This gives the outgoing jet a transverse momentum kick of the order of the $W$ boson mass. The fact that the invariant mass of the heavy system $X_{M}=t j$, as it appears in Eq. (9), is easily twice the threshold mass again contributes to the larger values of $Q_{b}^{\max }$. Finally, as discussed in the context of neutral Higgs bosons, production away from threshold lifts the degeneracy 

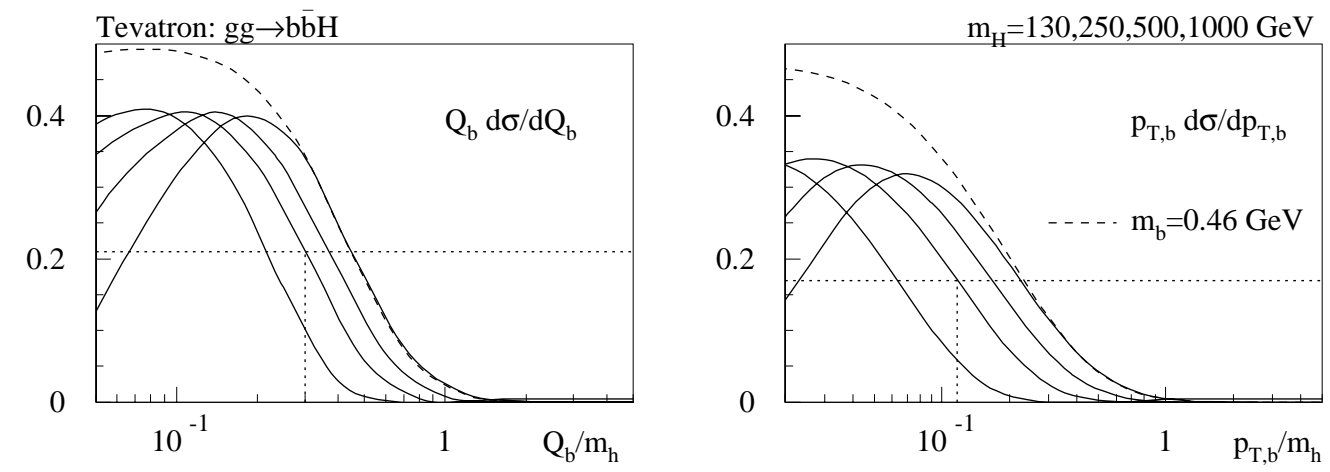

FIG. 9. Normalized distributions for the hadronic neutral Higgs boson production at the Tevatron. The normalization for the largest Higgs boson mass is by the total rate; for all other masses the curves are normalized such that the virtuality distributions coincide at their maxima. The normalization factors for the virtuality and the transverse momentum are identical. The dashed curve uses a mathematical cutoff $0.46 \mathrm{GeV}$ for the bottom quark mass and $130 \mathrm{GeV}$ for the Higgs boson mass. It is normalized to match the curve for the physical bottom quark mass for large virtuality. The curves are ordered on their downward slopes by decreasing final state mass toward larger values of $Q_{b}^{\text {max }}$ and $p_{T, b}^{\max }$.

of different values of $Q_{b}^{\max } / M$, pushing the lighter states to higher values of $Q_{b}^{\max }$.

We will not discuss the transition from the bottom quark virtuality to the transverse momentum in any detail. From Fig. 8 we see that the plateau is softened and $p_{T, b}^{\max }$ $\sim Q_{b}^{\max } / 2$, as in all other processes discussed before: the interpolation argument presented in Sec. IV describes the single top quark production perfectly well. This part of our argument indeed holds independently for all processes considered in this paper.

Recently, a similar issue of bottom partons was discussed [32] in the framework of single top quark production at a linear collider $e \gamma \rightarrow \bar{\nu} t \bar{b}$. The authors find sizable differences between the finite $m_{b}$ prediction and the (massless) structure function approach, predominantly close to threshold $\sqrt{s}$ $\lesssim m_{t}+m_{b}+10 \mathrm{GeV}$. These differences can in part be traced back to phase space effects. From Fig. 1 and Fig. 7 we see that this region of phase space contributes little to the Higgs boson sample at the LHC, after we convolute the partonic cross section with the gluon densities, integrating over the entire partonic energy range. It will have even less impact on the total rate once a minimum transverse momentum of the Higgs boson decay products is required. While for a linear collider the bottom quark mass is an important source of theoretical uncertainty and the collinear logarithms (multiplied with $\alpha \sim 1 / 137$ ) are under control, the dominant problem at hadron colliders is the size of the logarithms (multiplied with $\alpha_{s}$ ), which we link to the transverse momentum spectrum in the exclusive processes.

\section{CONCLUSIONS}

Starting from charged Higgs boson production in association with a top quark, we have investigated processes which can be described using bottom partons. From the kinematics of the exclusive processes, we find numerically that the factorization scale of the bottom parton has to be smaller than the threshold mass or the hard scale in the process: $\mu_{F, b}$ $\sim M / 5$. In two steps we first investigate the validity of the asymptotic approximation in the bottom quark virtuality and then in the transverse momentum. The upper limit $p_{T, b}^{\max }$, for which the exclusive cross section is dominated by collinear bottom quarks and large logarithms $\log \left(p_{T, b}^{2} / m_{b}^{2}\right)$, defines the appropriate value for the factorization scale of the bottom parton in the inclusive process. We derive the observed dramatic decrease in the factorization scale as compared to the hard scale $M$ in a process-independent approach, using only properties of the phase space and of the parton densities. In this simple picture we indeed find $\mu_{F, b} \equiv p_{T, b}^{\max } \sim M / 4$.

This choice of appropriate scales resolves the puzzle of the discrepancy between inclusive and exclusive rates, as has been presented in the literature. Using an appropriate scale, the difference for example for the process $b \bar{b} \rightarrow \Phi[9]$ is not huge and is well understood. Moreover, the higher order calculation of the process $b \bar{b} \rightarrow \Phi[10]$ shows an entirely flat scale dependence if one picks $\mu_{F, b} \sim M / 4$ as the central scale. We understand how this is caused by the partonic phase space and independently confirm these higher order results.

Turning around the argument, we can specify how large the logarithms actually are that are resummed in the bottom parton picture. Again, they are smaller than the naive guess $\log \left(M^{2} / m_{b}^{2}\right)$ would indicate. In particular, for a light neutral Higgs boson one can debate using the (resummed) bottom parton cross section, or just integrating over the exclusive cross section [33]. For heavier neutral or charged Higgs bosons the logarithms are certainly large enough to require a resummation.

In our chain of arguments we find a significant difference between the extent of the asymptotic behavior in terms of the intermediate bottom quark virtuality and the final state bottom quark transverse momentum-while naively both of these pictures should be equivalent. However, we find $p_{T, b}^{\max }$ $\sim Q_{b}^{\max } / 2$ and a visibly softened upper edge of the plateau in $p_{T, b} d \sigma / d p T, b$. Both effects can be traced back to the partonic phase space. Because factorization is shown in terms of the virtuality and final state logarithms and parton densities usually refer to the transverse momentum picture, these two 
approaches and the corresponding definitions of the collinear region of phase space leave an unsolved problem, which we treat as a theoretical uncertainty in the computation of $\mu_{F, b}$.

\section{ACKNOWLEDGMENTS}

We would like to thank Michael Krämer, Scott Willenbrock, and Fabio Maltoni for fruitful discussions. Moreover, we would like to thank Thomas Gehrmann, Dave Rainwater, and Robert Harlander for very helpful comments and the careful reading of the manuscript. T.P. would like to thank the Physics Department of the University of Edinburgh, where part of this work was completed, for their hospitality. E.B. was partly supported by the grants INTAS 00-0679, CERN-INTAS 99-377, and Universities of Russia UR.02.03.002.
[1] LEPEWWG Collaboration, D. Abbaneo et al., hep-ex/0212036.

[2] Z. Kunszt and F. Zwirner, Nucl. Phys. B385, 3 (1992); ATLAS TDR Collaboration, Report No. CERN/LHCC/99-15, 1999; CMS TP Collaboration, Report No. CERN/LHCC/94-38, 1994; M. Spira, Fortschr. Phys. 46, 203 (1998); M. Dittmar and H.K. Dreiner, Phys. Rev. D 55, 167 (1997); T. Plehn, D. Rainwater, and D. Zeppenfeld, ibid. 61, 093005 (2000); N. Kauer, T. Plehn, D. Rainwater, and D. Zeppenfeld, Phys. Lett. B 503, 113 (2001); V. Drollinger, T. Müller, and D. Denegri, hep-ph/0111312.

[3] T. Plehn, D. Rainwater, and D. Zeppenfeld, Phys. Lett. B 454, 297 (1999).

[4] ECFA/DESY LC Physics Working Group Collaboration, E. Accomando et al., Phys. Rep. 299, 1 (1998); ECFA/DESY LC Physics Working Group Collaboration, J.A. Aguilar-Saavedra et al., TESLA TDR, Report No. DESY-2001-011C.

[5] A. Dedes, S. Heinemeyer, S. Su, and G. Weiglein, hep-ph/0302174.

[6] J.F. Gunion, H.E. Haber, F.E. Paige, W.K. Tung, and S.S. Willenbrock, Nucl. Phys. B294, 621 (1987); D.A. Dicus, J.L. Hewett, C. Kao, and T.G. Rizzo, Phys. Rev. D 40, 787 (1989); W. Hollik and S.H. Zhu, ibid. 65, 075015 (2002).

[7] S.H. Zhu, hep-ph/0109269; Phys. Rev. D 67, 075006 (2003).

[8] T. Plehn, Phys. Rev. D 67, 014018 (2003).

[9] F. Maltoni, Z. Sullivan, and S. Willenbrock, Phys. Rev. D 67, 093005 (2003).

[10] R.V. Harlander and W.B. Kilgore, Phys. Rev. D 68, 013001 (2003).

[11] J.C. Collins and W.K. Tung, Nucl. Phys. B278, 934 (1986); M.A. Aivazis, J.C. Collins, F.I. Olness, and W.K. Tung, Phys. Rev. D 50, 3102 (1994); F.I. Olness and W.K. Tung, Nucl. Phys. B308, 813 (1988); R.M. Barnett, H.E. Haber, and D.E. Soper, ibid. B306, 697 (1988); J.C. Collins, Phys. Rev. D 58, 094002 (1998); M. Krämer, F.I. Olness, and D.E. Soper, ibid. 62, 096007 (2000).

[12] E. Eichten, I. Hinchliffe, K.D. Lane, and C. Quigg, Rev. Mod. Phys. 56, 579 (1984); 58, 1065 (1986); N.G. Deshpande, X. Tata, and D.A. Dicus, Phys. Rev. D 29, 1527 (1984).

[13] S.S. Willenbrock, Phys. Rev. D 35, 173 (1987); A. Krause, T. Plehn, M. Spira, and P.M. Zerwas, Nucl. Phys. B519, 85 (1998); S.H. Zhu, C.S. Li, and C.S. Gao, Phys. Rev. D 58, 055007 (1998); O. Brein and W. Hollik, Eur. Phys. J. C 13, 175 (2000); O. Brein, W. Hollik, and S. Kanemura, Phys. Rev. D 63, 095001 (2001).

[14] D.A. Dicus and C. Kao, Phys. Rev. D 41, 832 (1990); A.A. Barrientos Bendezu and B.A. Kniehl, ibid. 59, 015009 (1999);
63, 015009 (2001); S. Moretti and K. Odagiri, ibid. 59, 055008 (1999).

[15] A.C. Bawa, C.S. Kim, and A.D. Martin, Z. Phys. C 47, 75 (1990); F. Borzumati, J.L. Kneur, and N. Polonsky, Phys. Rev. D 60, 115011 (1999); A. Belyaev, D. Garcia, J. Guasch, and J. Sola, J. High Energy Phys. 06, 059 (2002).

[16] L.G. Jin, C.S. Li, R.J. Oakes, and S.H. Zhu, Eur. Phys. J. C 14, 91 (2000).

[17] M. Drees and D.P. Roy, Phys. Lett. B 269, 155 (1991); D.P. Roy, ibid. 283, 403 (1992); K. Odagiri, ibid. 452, 327 (1999); S. Moretti and D.P. Roy, ibid. 470, 209 (1999).

[18] D.P. Roy, Phys. Lett. B 277, 183 (1992); 459, 607 (1999).

[19] K.A. Assamagan and Y. Coadou, Acta Phys. Pol. B 33, 707 (2002); K.A. Assamagan, Y. Coadou, and A. Deandrea, EPJdirect 4, 9 (2002).

[20] D. Denegri et al. Report No. CMS-NOTE-2001-032, hep-ph/0112045; P. Salmi, R. Kinnunen, and N. Stepanov, hep-ph/0301166.

[21] M. Spira, A. Djouadi, D. Graudenz, and P.M. Zerwas, Nucl. Phys. B453, 17 (1995); R.V. Harlander and W.B. Kilgore, Phys. Rev. Lett. 88, 201801 (2002); C. Anastasiou and K. Melnikov, Nucl. Phys. B646, 220 (2002); V. Ravindran, J. Smith, and W.L. van Neerven, hep-ph/0302135.

[22] J. Dai, J.F. Gunion, and R. Vega, Phys. Lett. B 345, 29 (1995); D. Froidevaux and E. Richter-Was, Z. Phys. C 67, 213 (1995).

[23] D.A. Dicus and S. Willenbrock, Phys. Rev. D 39, 751 (1989); D. Dicus, T. Stelzer, Z. Sullivan, and S. Willenbrock, ibid. 59, 094016 (1999).

[24] R.K. Ellis, I. Hinchliffe, M. Soldate, and J.J. van der Bij, Nucl. Phys. B297, 221 (1988); C. Kao and N. Stepanov, Phys. Rev. D 52, 5025 (1995).

[25] E. Boos, A. Djouadi, M. Mühlleitner, and A. Vologdin, Phys. Rev. D 66, 055004 (2002).

[26] E. Boos, A. Djouadi, and A. Nikitenko, hep-ph/0307079.

[27] J. Campbell, R.K. Ellis, F. Maltoni, and S. Willenbrock, Phys. Rev. D 67, 095002 (2003).

[28] D. Rainwater, M. Spira, and D. Zeppenfeld, hep-ph/0203187.

[29] D. Stump, J. Huston, J. Pumplin, W.K. Tung, H.L. Lai, S. Kuhlmann, and J.F. Owens, hep-ph/0303013.

[30] A.P. Heinson, A.S. Belyaev, and E.E. Boos, Phys. Rev. D 56, 3114 (1997); A.S. Belyaev, E.E. Boos, and L.V. Dudko, ibid. 59, 075001 (1999).

[31] T. Stelzer, Z. Sullivan, and S. Willenbrock, Phys. Rev. D 56, 5919 (1997); B.W. Harris, E. Laenen, L. Phaf, Z. Sullivan, and S. Weinzierl, ibid. 66, 054024 (2002).

[32] J.H. Kühn, C. Sturm, and P. Uwer, hep-ph/0303233.

[33] W. Beenakker, S. Dittmaier, M. Krämer, B. Plümper, M. Spira, and P.M. Zerwas, Phys. Rev. Lett. 87, 201805 (2001); Nucl. Phys. B653, 151 (2003); S. Dawson, L.H. Orr, L. Reina, and D. Wackeroth, Phys. Rev. D 67, 071503(R) (2003). 